\title{
Método de Montecarlo como estrategia didáctica intercultural para la enseñanza universitaria de la física y matemática en el contexto de la educación no presencial
}

\section{Monte Carlo method as an intercultural didactic strategy for university teaching of Physics and Mathematics in the context of non-face-to-face education}

\author{
Ronald Omar Estela Urbina, ${ }^{1 a}$ Elisa Contreras Barsallo, ${ }^{2}$ Fernando Alain Incio Flores, ${ }^{3}$ \\ Roger Álvaro Fernández Villarroel, ${ }^{4}$ José Orlando Namuche Paiva, ${ }^{5}$ \\ Edinson Enrique Reyes Alva ${ }^{6}$ \\ Universidad Nacional Intercultural Fabiola Salazar Leguía de Bagua, Peru ${ }^{1}$ \\ I.E Túpac Amaru de Chiriaco-Imaza-Amazonas, Perú ${ }^{2}$ \\ Universidad Cesar Vallejo, Lima, Perú ${ }^{3}$ \\ Universidad Nacional Intercultural Fabiola Salazar Leguía de Bagua, Perú ${ }^{4}$ \\ I. E María Auxiliadora de Chachapoyas-Amazonas ${ }^{5}$ \\ Universidad Nacional Toribio Rodríguez de Mendoza, Chachapoyas, Perú ${ }^{6}$
}
ORCID ID: $\underline{\text { https://orcid.org/0000-0001-5240-1242 }}{ }^{1}$
ORCID ID: https://orcid.org/0000-0002-0278-7252 2
ORCID ID: $\underline{\text { https://orcid.org/0000-0003-3286-7787 }}{ }^{3}$
ORCID ID: https://orcid.org/0000-0001-7912-7722
ORCID ID: https://orcid.org/0000-0001-8778-0094
ORCID ID: $\underline{\text { https://orcid.org/0000-0001-9529-3667 }}{ }^{6}$

Recibido: 15 de febrero de 2021

Aceptado: 14 de mayo de 2021

\section{Resumen}

La educación universitaria intercultural enfrenta actualmente desafíos en el sistema educativo debido a la pandemia del covid-19. El reto para el docente como "mediador" es innovar diversas estrategias para socializar los saberes comunes y el conocimiento ancestral entre los estudiantes originarios y mestizos en entornos no presenciales dentro de un clima de tolerancia, diálogo y respeto cultural. El presente artículo tiene como objetivo socializar de manera virtual las prácticas culturales y el conocimiento ancestral de los estudiantes Awajun y Wampis en la carrera de ingeniería civil. La propuesta consiste en actividades virtuales que son simulaciones numéricas

${ }^{\mathrm{a} C}$ Correspondencia al autor:

E-mail: restela@unibagua.edu.pe 
aplicando el método Montecarlo, cuya finalidad es estimar numéricamente una variable, y de esa forma comprobar un principio físico-matemático empleando materiales de su comunidad. La presente innovación pedagógica responde a una investigación documental descriptiva, cuya metodología consistió en tomar como referencia trabajos de investigación realizados entre los años 2019, 2020 y 2021 referidos a la educación intercultural utilizando estrategias lúdicas en el aula; así mismo se desarrolló una actividad virtual que los estudiantes replicaron de forma contextualizada evidenciando el saber sistematizado con enfoque intercultural.

Palabras claves: método, intercultural, Montecarlo, simulación.

\begin{abstract}
Intercultural university education is currently facing challenges in the educational system due to the covid-19 pandemic. The challenge for the teacher as a "mediator" is to innovate various strategies to socialize common knowledge and ancestral knowledge among native and mixed-race students in non-contact settings within a climate of tolerance, dialogue and cultural respect. The objective of this article is to socialize in a virtual way the cultural practices and ancestral knowledge of the Awajun and Wampis students in the civil engineering career. The proposal consists of virtual activities that are numerical simulations applying the Monte Carlo method, whose purpose is to numerically estimate a variable, and thus verify a physical-mathematical principle using materials from their community. The present pedagogical innovation responds to a descriptive documentary research, whose methodology consisted of taking as a reference research works carried out between 2019, 2020 and 2021 referring to intercultural education using ludic strategies in the classroom; Likewise, a virtual activity was developed that the students replicated in a contextualized way, evidencing the systematized knowledge with an intercultural approach.
\end{abstract}

Keywords: strategy, intercultural, Monte Carlo, ancestral knowledge.

\title{
Introducción
}

La actual emergencia sanitaria decretada a nivel mundial constituye un desafío no solo en el sector salud y económico en Latinoamérica, sino también un reto del sector educativo, que busca masificar el conocimiento a través de diversas herramientas tecnológicas. Esta realidad representa una oportunidad para reflexionar sobre el proceso de aprendizaje de los estudiantes y replantear una propuesta innovadora en la práctica profesional en las universidades (Ruíz, 2020). Es por ello que la educación de calidad es un proceso constante de transformación, retos y desafíos. En este proceso de cambio, la educación universitaria no presencial que se está desarrollando actualmente en el Perú ha marcado nuevas políticas y directivas de emergencia en nuestro sistema educativo en general. Ante este contexto, la utilización de una didáctica innovadora y pertinente permitirá la adquisición de nuevas experiencias de aprendizaje contextualizado, significativo y con sentido para los estudiantes. 
La utilización de una didáctica innovadora y pertinente en una educación virtual o no presencial permite no solo nuevas formas de enseñar, sino creativas formas de aprender en un contexto donde cobra relevancia la capacidad de gestionar el aprendizaje autónomo de los estudiantes y autoevaluación constante de sus aprendizajes logrados. La selección pertinente del campo temáticos con sus respectivas competencias, y la estrategia para innovar, organizar tiempos de acuerdo a las necesidades de los estudiantes, como el caso de la conectividad, uno de las dificultades más recurrentes en los estudiantes contribuyen a generar un ambiente de aprendizaje más contextualizado, pertinente y significativo (Cifuentes, 2020).

\section{Método de Montecarlo como estrategia didáctica}

También llamado Simulación de Montecarlo se define como un procedimiento matemático que puede ser automatizado por medio de un computador, con la finalidad de proporcionar "datos" de un muestreo de tipo aleatorio tomando como referencia distribuciones ya conocidas y utilizarlos para realizar "experimentos" numéricos. Esta simulación numérica resulta muy útil, ya que puede usarse en aquellas situaciones cuando se quiere hacer estimaciones numéricas; así como tomar decisiones sobre variables inciertas. También se presentan algunas desventajas, pues se tiene que disponer de mucho tiempo para el cálculo cuando se requiere un número elevado de eventos aleatorios para acercarse lo más posible a los valores reales (Mera, Otero, \& Calle, 2021).

La simulación numérica de Montecarlo permite replicar un evento aleatorio, es decir tenemos la posibilidad de múltiples resultados con el mismo valor de entrada de datos. Este método es muy aplicable en la matemática, y en particular se utilizan en la resolución de problemas geométricos donde se han podido estimar áreas de superficies en base a esta simulación. La cercanía de la respuesta en comparación con el valor real o teórico dependerá de la repitencia de los eventos, esto significa que a mayor número de eventos se espera más finos y mejores resultados. Asimismo este método puede realizarse manualmente, sin embargo una limitante sería el número elevado de eventos que se puedan realizar, por ello una alternativa para optimizar los resultados sería la simulación asistida por computador (Prieto \& Cabrera, 2020).

Los simuladores como estrategia didáctica para la enseñanza de la física y matemática permiten establecer un nexo entre el componente teórico de la asignatura y su correspondiente complemento práctico como lo indica la figura 1. En condiciones normales de enseñanza presencial 
se podría acceder a los laboratorios de la universidad; sin embargo hoy en día, donde se vive actualmente el distanciamiento social obligatorio como protocolo de sanidad pública, el laboratorio virtual cobra más vigencia que nunca, donde en cada aula virtual se evidencia aprendizajes significativos en un contexto lúdico (Estela, Contreras, \& Incio, 2020).

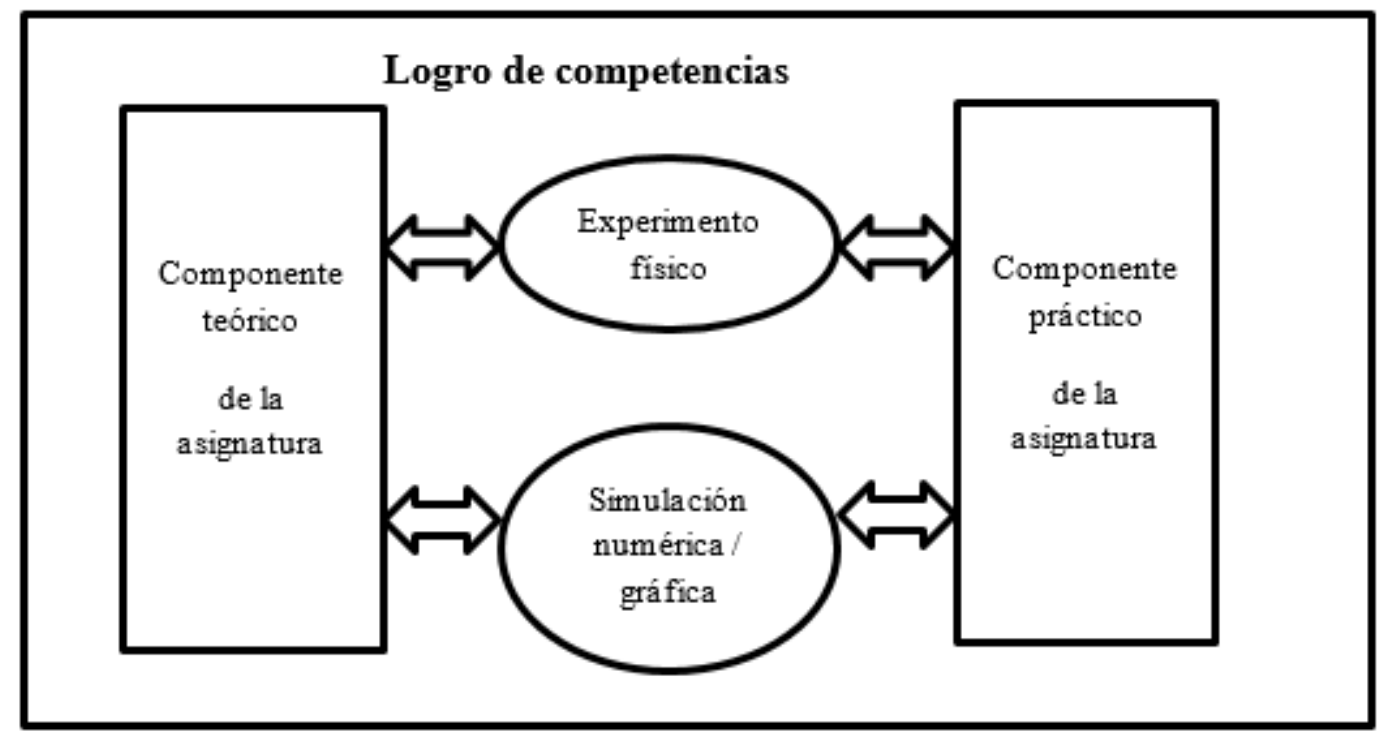

Figura 1. Logro de competencias curriculares a través de las asignaturas Fuente: Elaboración propia

La simulación numérica o gráfica vista como una estrategia didáctica no es una actividad restrictiva o limitante en la innovación de los agentes educativos; todo lo contrario, debe entenderse como un juego o actividad lúdica donde el componente sorpresa o aleatorio debe ser predominante. El juego genera en el estudiante una actitud de superación a vencer o superar ciertos niveles o estándares establecidos en estas aplicaciones informáticas, y si este juego se liga con un campo temático de matemática o física, generan en el estudiantes aprendizajes significativos en entornos amigables (Pamplona, Cuesta, \& Cano, 2019).

\section{Interculturalidad en la virtualidad}

El Perú es un país pluricultural y multilingüista donde convergen diversas etnias con su respectiva cosmovisión. De esta manera, las aulas universitarias no están exentas a esta realidad. Sin embargo, cabe recalcar que muchas veces las definiciones de pluriculturalidad y multiculturalidad suelen ser asociados de manera automática a la interculturalidad. De forma general podríamos decir que, la pluriculturalidad es la evidencia de la presencia de dos o más 
culturas en un mismo espacio; por otro lado, la multiculturalidad representaría la aceptación explícita de esta existencia en forma simultánea. Concluyentemente podríamos decir que estas dos definiciones son los supuestos para que la interculturalidad se evidencie (Carrillo, 2020).

El sistema educativo en el nivel superior busca desarrollar diversas competencias, habilidades y desempeños en los estudiantes que le permita insertarse exitosamente al campo laboral. Para este fin es necesario centrarse en el aprendizaje de los estudiantes, siendo de gran importancia el desarrollar diversas estrategias por parte del docente para lograr afianzar estos aprendizajes. Así como fortalecer y fomentar la interculturalidad en el aula tanto en su modalidad presencial como virtual. De esta manera, el docente se convierte en un "mediador cultural" es decir representa el nexo entre las culturas intervinientes en nuestros espacios educativos (Ruiz-Quiroga, 2020).

La visión de la diversidad cultural está presente en las aulas. Algunos estudiantes son conscientes de esta realidad y reafirman su identidad a través de la revaloración de su cultura; otros por vergüenza o timidez deciden alejarse de sus prácticas ancestrales. Sin embargo, surge la necesidad de empoderar a los estudiantes en el reconocimiento y aceptación de su identidad cultural. Por ello, el trabajo intercultural debe afianzar la relación entre culturas, donde no solo se muestre su interés como una realidad lejana, mítica, sino al contrario una cultura viva, enriquecedora en sus prácticas tradicionales (Guajardo, 2020).

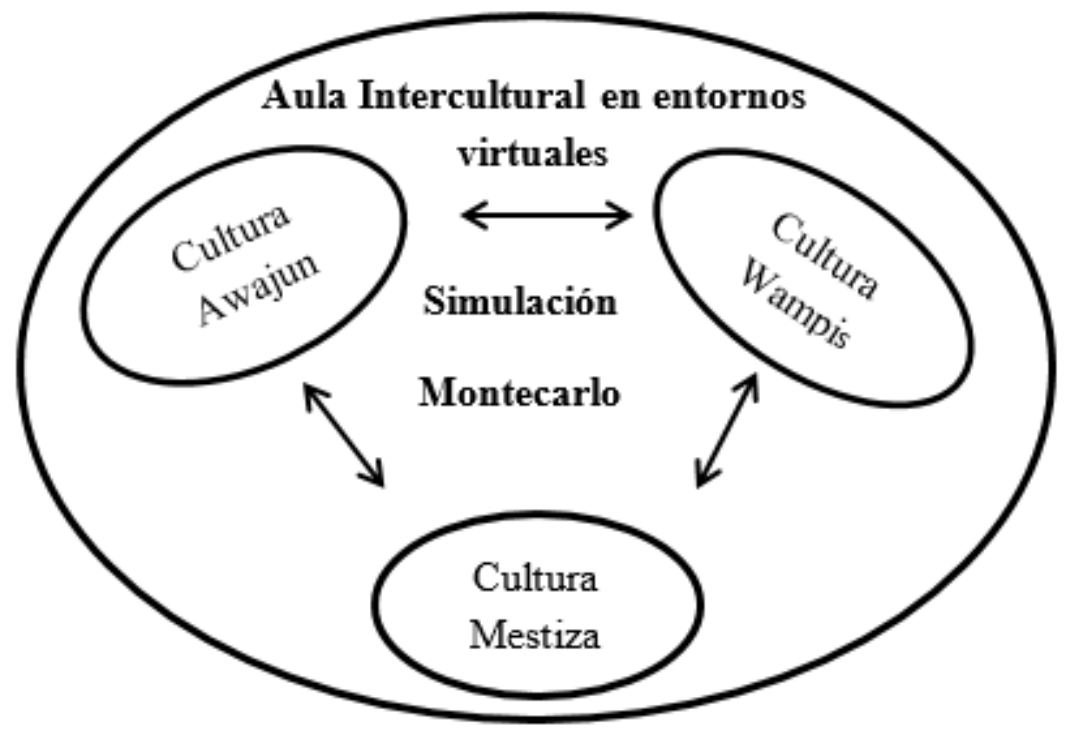

Figura 2. Aula virtual en entornos interculturales Fuente: Elaboración propia 
El docente universitario intercultural debe estar capacitado para propiciar innovaciones e investigaciones educativas para convertir el aula en un "laboratorio" donde el docente se encuentra retado constantemente a experimentar estrategias didácticas que permitan despertar el interés cultural y la tolerancia entre las culturas intervinientes (Alonso, Roque, \& Juarez, 2019).

El desarrollo de competencias es inherente a la educación universitaria. Todo esto será posible en la medida que el estudiante a través del conocimiento desarrolle habilidades de investigación que no solo se circunscriba a la repitencia de cierto conocimiento, sino a cómo socializar o comunicar el aprendizaje contextualizado, y ser capaz de transformarlo en nuevas propuestas para la solución de problemas. En este escenario juegan un papel muy importante las actividades lúdicas que permitan al estudiante elegir la más pertinente entre múltiples opciones, luego socializar sus prácticas en base a patrones culturales a partir del conocimiento común o cotidiano (Estela et al., 2020).

En la actualidad la educación universitaria virtual en el sistema peruano surge como una respuesta a la emergencia sanitaria debido al COVID-19. Esta realidad nos muestra que no nos encontrábamos preparados para afrontar la enseñanza virtual, pues los docentes, en su gran mayoría carecían de experiencia en entornos virtuales. No obstante existió mucha inversión de parte de las instituciones educativas para dotar a sus docentes en el conocimiento y uso de herramientas digitales y así afrontar con éxito la adaptación de nuestra aula virtual tradicional en un aula virtual intercultural (Amaya-Amaya, Cantú-Cervantes, \& Marreros-Vázquez, 2021).

A la luz de este contexto, el objetivo de esta investigación es socializar una estrategia didáctica intercultural basada en simulaciones numéricas del método Montecarlo, donde los estudiantes desarrollaron actividades lúdicas, fundamentados en principios físicos-matemáticos para determinar una variable por métodos estadísticos aleatorios.

\section{Metodología}

La presente innovación pedagógica como propuesta responde a una investigación documental descriptiva. La muestra fue constituida por publicaciones de investigaciones de los años comprendidos entre 2019-2021 referidos a la educación intercultural, así como recolección de saberes ancestrales awajún-wampis por parte de los estudiantes de la UNIFSLB como parte de su experiencia vivencial en sus comunidades o entornos inmediatos. Los datos se recogieron utilizando la técnica de revisión de documentos (Alonso et al., 2019). 
Esta propuesta didáctica es una mixtura entre la documentación revisada, así como la información de primera mano que representa la experiencia del trabajo intercultural (Antioquia, 2019) como docentes de los cursos de Física y Matemática en las aulas universitarias de la UNIFSLB, donde su población estudiantil es $50 \%$ originario (provenientes de las comunidades Awajun y Wampis de la amazonia peruana) y el otro 50\% estudiantes mestizos o hispanohablantes de las zonas principalmente de Bagua capital y Utcubamba.

Esta propuesta desarrolló una serie de acciones o pasos para su ejecución. Como primer paso es la elección de un campo temático en los cursos seleccionados: cálculo de centroides en elementos geométricos correspondiente al tema de momento de inercia para la estructura curricular del curso de Estática y Dinámica, así también para modelar numéricamente algunos productos de la zona de Bagua en el curso de Métodos Numéricos en el caso de la carrera profesional de ingeniería civil.

Luego se realizó un taller de capacitación en herramientas informáticas, específicamente en elaboración de algoritmos y lenguaje de programación Basic, orientado a los estudiantes para que puedan afrontar con éxito la puesta en marcha de sus programas informáticos. Asimismo se desarrolló el algoritmo de las cuatro actividades didácticas interculturales que se presentan como propuesta a los estudiantes, permitiéndoles iniciar la aplicación del método Montecarlo de forma manual, es decir cada estudiante dejará caer elementos pequeños (granos de arroz) sobre una superficie.

Se estableció la ruta de trabajo, brindando recomendaciones a todos los estudiantes sobre la estructura de la experiencia de aprendizaje, así como la rúbrica de evaluación, donde se ponderaba el componente cultural en la aplicación realizada, como indica la figura 3. De la misma forma se incide en la utilización de materiales de la zona, sobre todo de las comunidades originarias ya sea Wampis o Awajun.

Posteriormente se socializó la aplicación del método Montecarlo vía Google Meet, a través de una videoconferencia. Cada estudiante expuso una de las cuatro actividades planteadas al inicio de esta experiencia de aprendizaje. Durante la sesión virtual sincrónica, el docente procedió a evaluar las exposiciones sobre la aplicación del método Montecarlo por medio de una rúbrica previamente socializada a los estudiantes. 


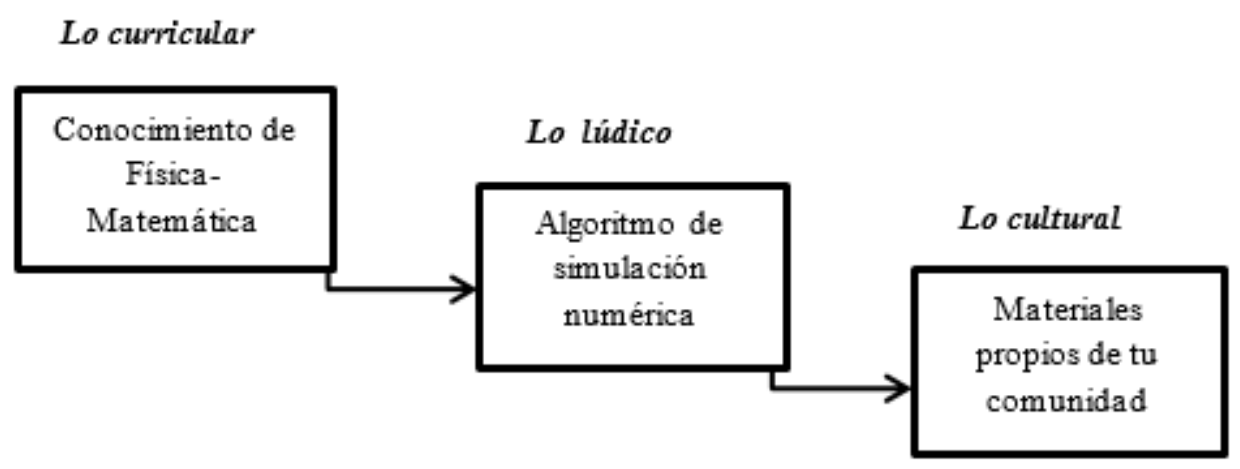

Figura 3. Simulación como estrategia intercultural Fuente: Elaboración propia

\section{Resultados}

A continuación, se muestra una de las cuatro actividades que conforman las estrategias didácticas interculturales propuestas en la Universidad Nacional Intercultural "Fabiola Salazar Leguía” de Bagua, diseñada para la estructura curricular del curso de Estática y dinámica en la carrera profesional de Ingeniería civil. La estructura de cada actividad posee un nombre, siendo su característica principal inclusiva, además de referenciar materiales o elementos de la región Amazonas. El segundo componente de la actividad es el conocimiento sistematizado (Currículo); el tercer componente, Método Montecarlo (Lúdico), y por último los Elementos de tu comunidad (Cultura).

\section{Actividad 1}

Nombre: Estimando el espesor de la caña de Bambú

Objetivo: Estimar numéricamente el espesor o grosor de una caña de bambú (Guayaquil) empleando el método aleatorio de Montecarlo de modalidad manual y computarizada.

\section{Conocimiento sistematizado (Currículo)}

El área de un círculo es la cantidad de espacio que contiene o lo abarca. De otra forma podemos definirla como la cantidad total de espacio dentro del círculo.

$$
A_{c}=\int_{0}^{2 \pi} \int_{0}^{r} \operatorname{rdrd} \theta
$$


Para calcular el área de un círculo haremos uso de la siguiente fórmula:

Área de un círculo $\mathrm{A}_{\mathrm{c}}=\pi \mathrm{r}^{2}$

Área de la corona circular $A_{c}=\pi\left(R^{2}-r^{2}\right)$

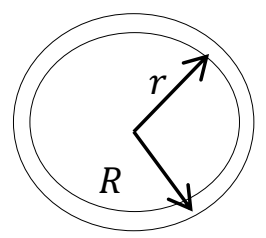

\section{Método Montecarlo (lúdico)}

En primer momento lo haremos de forma manual: Al verter pequeños elementos, por ejemplo, granos de arroz sin cáscara sobre la sección transversal de la caña de Guayaquil podremos contabilizar manualmente cuántos cayeron sobre el espesor o pared. Sin embargo, debemos advertir que para obtener una estimación más próxima al valor real, es necesario utilizar elementos más pequeños que los granos de arroz que logren una mayor cobertura de área, por ello se propone utilizar grageas, pero siendo estos pequeños confites de mayor dificultad para ser contabilizados, y de poco acceso para adquirirlas en las comunidades originarias amazónicas, se sugiere la utilización de los granos de arroz que ellos cultivan.

$N_{\text {total }}=$ Número total de granos de arroz que se vierten

$N_{\text {grosor }}=$ Número de granos de arroz que caen en el espesor del bambú

$$
\begin{aligned}
& \frac{N_{\text {grosor }}}{N_{\text {total }}}=\frac{\pi\left(R^{2}-r^{2}\right)}{\pi R^{2}} \\
& r=R \sqrt{\left(1-\frac{N_{\text {grosor }}}{N_{\text {total }}}\right)}
\end{aligned}
$$

Esta expresión matemática nos permitirá conocer el radio interno sin necesidad de cortar la caña de bambú conociendo previamente su perímetro. 


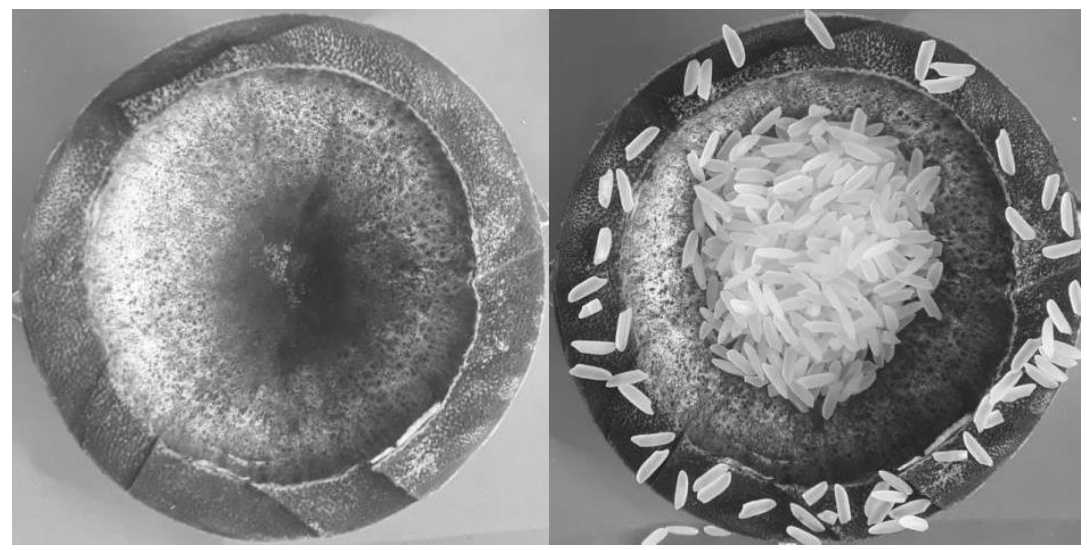

Figura 4. Método Montecarlo realizada de forma manual usando granos de arroz Fuente: Elaboración propia

Posteriormente se realizó el método Montecarlo de forma computarizada lo que nos permitirá tomar en cuenta valores más grandes de los números de puntos o eventos simulados: 


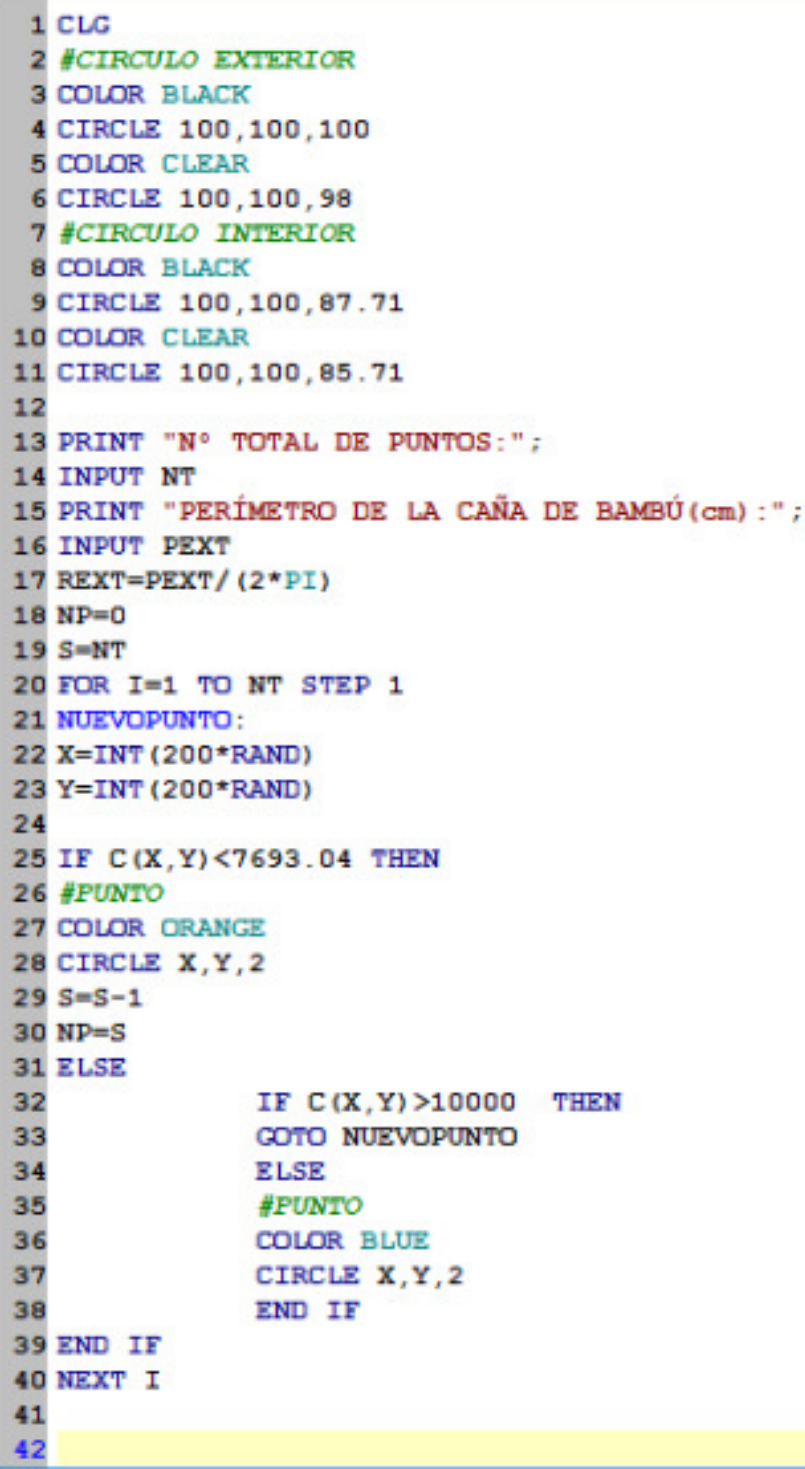



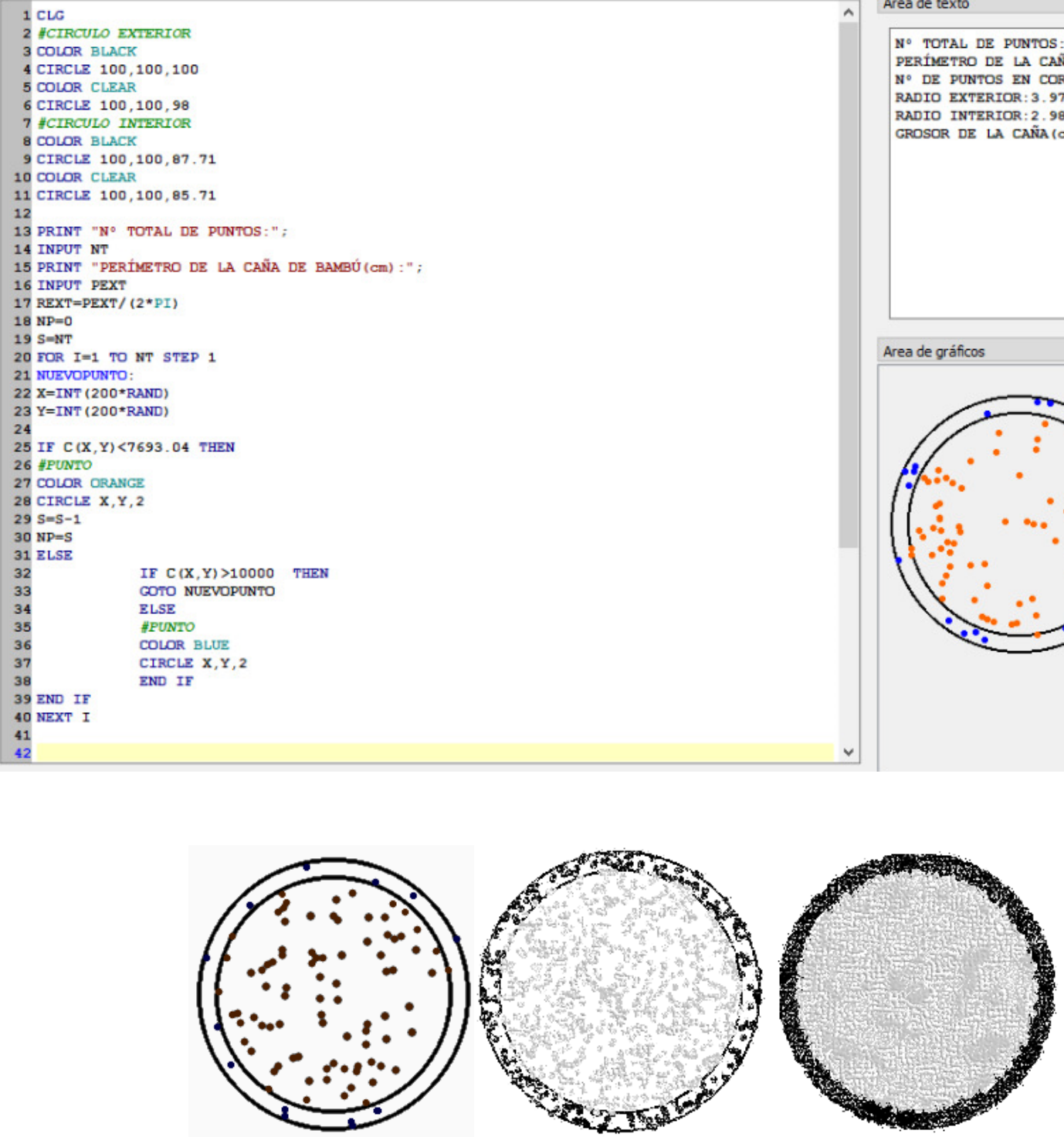

Figura 5. Método Montecarlo para 100, 1000 y 10000 eventos aleatorios computarizados Fuente: Elaboración propia

\section{Elementos de tu comunidad (cultura)}

Sin duda la caña bambú es, debido a estudios estructurales y térmicos, uno de los materiales más sostenibles que el hombre utiliza en la actualidad y se ha convertido en una alternativa predominante para el ámbito de la construcción y en particular, por el tipo de vivienda con un agradable confort (Pascual, 2008).

Desde lo estructural podemos afirmar que sus características les proporcionan muy buena resistencia con respecto a su inercia. Son de sección circular o aproximadamente circulares en su sección transversal, ordinariamente huecas en su interior, y con tabiques transversales de muy buena rigidez, colocados de tal forma para que no se provoque la ruptura al flexionarse. En esta 
ubicación pueden jugar un papel eficiente, proporcionándole resistencia mecánica y formando un firme y resistente armazón (Rodríguez, 2006).

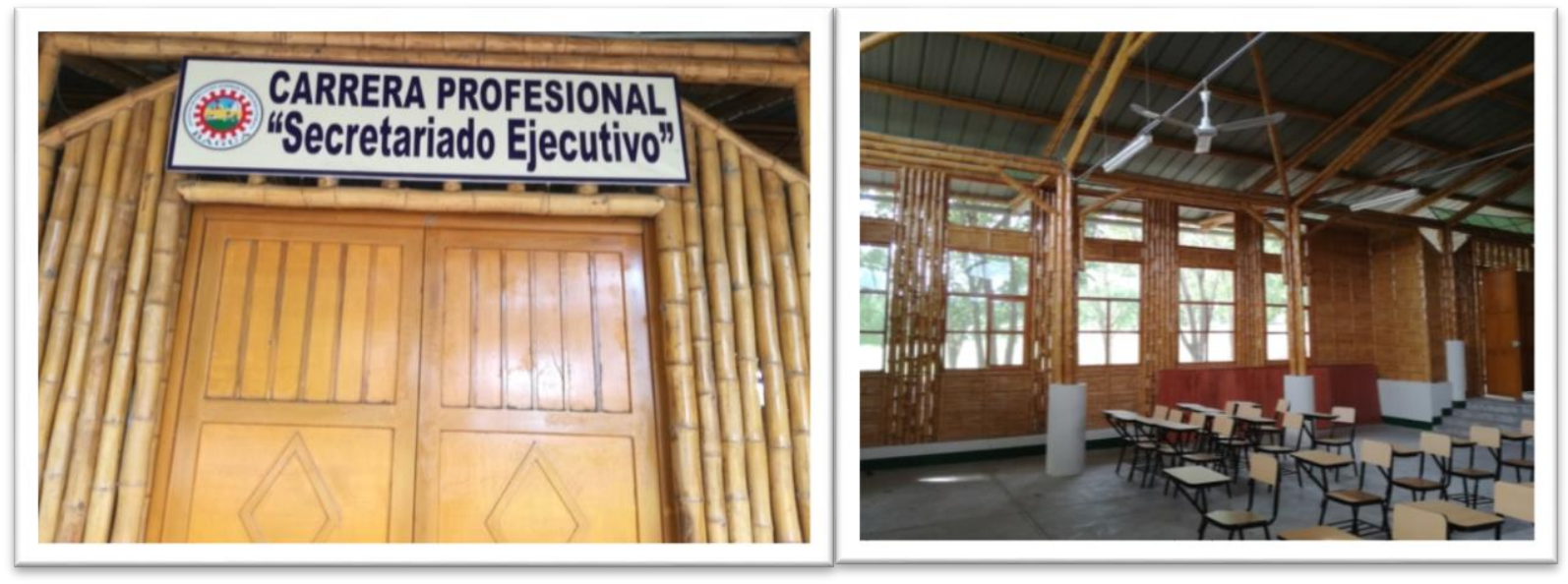

Figura 6. Uso de la caña de bambú en construcciones en la ciudad de Bagua-Amazonas-Perú. Instituto Tecnológico de Bagua.

Fuente: Elaboración propia

Cada actividad se consolida numéricamente completando la Tabla 1, donde el objetivo es la comparación numérica de los resultados obtenidos manualmente y de forma computarizada.

Tabla 1

Comparación porcentual del valor obtenido de manera manual y computarizada de la simulación Montecarlo.

\begin{tabular}{llll}
\hline $\begin{array}{l}\text { Nombre de la } \\
\text { actividad }\end{array}$ & $\begin{array}{l}\text { Valor del espesor } \\
\text { obtenido de la } \\
\text { simulación realizada } \\
\text { manualmente } \\
(\mathrm{cm})\end{array}$ & $\begin{array}{l}\text { Valor del espesor } \\
\text { obtenido de la } \\
\text { simulación } \\
\text { realizada con el } \\
\text { software }-100 \\
\text { veces } \\
(\mathrm{cm})\end{array}$ & $\begin{array}{l}\text { Valor del espesor } \\
\text { obtenido de la } \\
\text { simulación } \\
\text { realizada con el } \\
\text { software }-10000 \\
\text { veces } \\
(\mathrm{cm})\end{array}$ \\
\hline $\begin{array}{l}\text { Estimando el } \\
\text { espesor de la }\end{array}$ & & & \\
caña de & & 1.24 & 1.10 \\
Bambú & 0.818 & & \\
\hline
\end{tabular}


A continuación, mencionaremos las tres actividades restantes.

\section{Actividad 1}

Nombre: Estimando el área de mi terreno.

\section{Actividad 2}

Nombre: Estimando el volumen de la pulpa de coco.

\section{Actividad 3}

Nombre: Estimando la temperatura de equilibrio del jugo de aguaje.

\section{Discusión}

Esta propuesta didáctica intercultural plantea la utilización de herramientas digitales en la educación universitaria no presencial o virtual, Actualmente nuestro contexto está marcado por lo globalizado de las actividades, la incertidumbre y la era digital, incluyendo hoy en día el distanciamiento social obligatorio por la pandemia mundial. Esta realidad representa un sinnúmero de retos en todos los ámbitos de nuestro país, incluyendo al sistema educativo en general. Ante este escenario es pertinente que los docentes fortalezcan las competencias interculturales en espacios académicos entre sus colegas y los estudiantes (Pardo, Marín, \& De Castro, 2021).

Las estrategias de aprendizaje interculturales que proponen actividades lúdicas virtuales contribuyen a la adquisición de aprendizajes significativos en los estudiantes. Esta propuesta es factible, ya que los participantes agregan a estas actividades información, datos de su entorno más cercano contando con la presencia de la familia o de personajes ilustres de su comunidad que le permita mayor fiabilidad de su conocimiento (Ramírez, 2021). Las estrategias didácticas que incluyen actividades a través del juego y la experiencia de su entorno sirven de ente motivador para la participación activa de los estudiantes, y por ende la mejora de los aprendizajes (Parra, 2020).

Por otro lado, las actividades virtuales presentadas en este artículo deben ser adecuadas a ciertas características temáticas de los cursos de física o matemática, sin embargo pudiendo ser adecuadas a otras asignaturas como por ejemplo, comunicación, lengua nativa o historia que son los cursos que se imparten en los primeros ciclos como cursos generales de su formación profesional, es decir es labor del docente seleccionar adecuadamente los núcleos temáticos donde se puedan aplicar nuestra estrategia didáctica intercultural, esto conlleva a tener una posición crítica 
también respecto a la pertinencia curricular, es decir debe existir una análisis previo del colectivo docente respecto a la contextualización del currículo, y como esos contenidos resultan más cercanos al estudiante originario (Alarcón \& Márquez, 2019).

Una de las limitantes para el éxito de esta propuesta didáctica es el intercambio de diálogos entre los estudiantes, en especial entre los jóvenes originarios, quienes presentan dificultades para establecer relaciones interpersonales con sus compañeros, debido a la timidez, las diferentes prácticas culturales o la utilización de su misma lengua originaria. Es por ello, la importancia de establecer recursos y estrategias que permitan el intercambio de ambas culturas en un clima de igualdad y respeto cultural (Zavala \& Franco, 2020).

La precisión numérica del método Montecarlo es más óptima cuanto más eventos aleatorios se realicen, es decir si el estudiante realiza una simulación su margen de error o su dispersión respecto al valor real, será mayor, generando una necesidad de automatizar; es decir simular el proceso con mayor cantidad de eventos realizados en el computador o a través del software (Gómez, Cano, \& Montoya, 2020).

La educación intercultural en las últimas décadas se ha convertido en un fracaso porque no ha permitido disminuir la brecha de desigualdad en cuanto a políticas educativas que prevalezcan en el tiempo y no a cada gobierno vigente, así como la implementación de material educativo contextualizado a su lengua materna, docentes originarios e hispanos capacitados para innovar, investigar y aplicar estrategias interculturales que permita la conservación y revaloración de lenguas maternas, así como su conocimiento empírico en las diversas actividades que realiza convirtiéndose en prácticas ancestrales. La interculturalidad en espacios educativos debe permitir la coexistencia de la diversidad de etnias con sus costumbres y prácticas ancestrales, sin pretender subyugarla a la cultura hispana o en el peor de los casos aniquilarla. Por ello, el rol del docente como "mediador cultural" es de suma importancia y permitirá el éxito de la propuesta pedagógica, ya que permite el diálogo intercultural en las aulas sin discriminación (Bermejo, Maquera, \& Bermejo, 2020).

Ante la diversidad cultural de los estudiantes surge la necesidad de una propuesta didáctica intercultural pertinente, y su posterior implementación pedagógica; sin embargo, no debe ser una acción parcializada o focalizada de un curso universitario, todo lo contrario, debe ser el resultado del análisis colectivo de áreas curriculares, en ello radica su éxito. Una constante capacitación del docente contribuye a una adecuada formación intercultural, y permite generar espacios lúdicos de 
aprendizaje contextualizados entre estudiantes originarios e hispanos dentro de un ambiente de diálogo, tolerancia y respeto en el aula (Guajardo, 2020).

Los docentes universitarios intervienen y propician espacios de indagación e investigación para la mejora del proceso de enseñanza-aprendizaje en la modalidad no presencial o virtual. De esta manera garantiza la continuidad de los estudios en estos tiempos de aislamiento social obligatorio, donde se suma la correspondencia entre el contenido disciplinar y los elementos de su entorno (Bojórquez, 2020).

\section{Conclusiones}

La simulación numérica de Montecarlo en tiempos de educación no presencial, debido al confinamiento social obligatorio, ocasionado por la pandemia del Covid 19, resultó muy pertinente, ya que en la actualidad no se cuenta con acceso a laboratorios físicos en las universidades, por lo tanto, la utilización de la recreación o simulación virtual es una estrategia didáctica adecuada para generar aprendizajes significativos.

El aula virtual o presencial "tradicional" se puede convertir en un aula "intercultural" con la participación activa del docente como "mediador cultural”, para ello es necesario la capacitación, conocimiento de la cultura del entorno, innovación y apertura al diálogo por parte del docente para aplicar estrategias didácticas pertinentes y contextualizadas.

El método simulación de Montecarlo permitió realizar recreaciones lúdicas del entorno de los estudiantes; de esta manera, a través de espacios virtuales se socializó las prácticas culturales de los estudiantes originarios en un ambiente de tolerancia, diálogo y respeto cultural por parte de sus compañeros y docente. Asimismo, como estrategia didáctica intercultural resultó muy efectivo, ya que los aprendizajes que se propiciaron de manera lúdica se convirtieron en experiencias significativas, a través de un conocimiento cercano y contextualizado en el que los jóvenes lo diversifican de acuerdo a los materiales empleados.

La pertinencia cultural en la aplicación de este método radicó en que las actividades están ligadas a elementos cercanos a su quehacer cotidiano, al conocimiento empírico cultural que poseen, por ejemplo, resistencia de los materiales, el dominio de la flotación, lanzamiento de cuerpos, etc. que realizan en las comunidades Awajun y wampis. Estos elementos generaron interés y mayor participación de los estudiantes tanto originarios como hispanos. Por ello, el método Montecarlo como estrategia didáctica intercultural puede emplearse para la enseñanza de la física 
o la matemática superior, para ello no es necesario poseer conocimientos avanzados de la informática y estadística.

\section{Referencias}

Alarcón, J., \& Márquez, J. (2019). Competencias docentes interculturales. Multiculturalidad y consecuencias para la inmigración. Estudios Pedagógicos, 65 (2), 7-27. https://doi.org/10.4067/S0718-07052019000200007

Alonso, S., Roque, Y., \& Juarez, V. (2019). La educación intercultural en el contexto ecuatoriano de educación superior: un caso de innovación curricular. Tendencias Pedagógicas, 33, 4758. https://doi.org/10.15366/tp2019.33.004

Amaya Amaya, A., Cantú Cervantes, D., \& Marreros Vázquez, J. G. (2021). Análisis de las competencias didácticas virtuales en la impartición de clases universitarias en línea, durante contingencia del COVID-19. 21, 1-20.

Antioquia, D. (2019). Interculturalidad en el marco normativo de la educación física en Colombia. Introito a la formación profesional. Educación, 28 (54), 87-104. https://doi.org/doi.org/10.18800/educacion.201901.005

Bermejo, S., Maquera, Y., \& Bermejo, L. (2020). Procesos de educación intercultural y autosegregación indígena en los aimaras de Puno. Revista de Historia y Educación Latinoamericana, 22 (34), 19-43. https://doi.org/https://doi.org/10.19053/01227238.10101

Bojórquez, E. M. (2020). Percepciones de los estudiantes de la Universidad Autónoma Indígena de México en la modalidad virtual en tiempos del COVID-19. 47-61.

Cáceres, K. (2020). Educación virtual: Creando espacios afectivos, de convivencia y aprendizaje en tiempos de COVID-19. CienciAmérica, 9 (2), 38. https://doi.org/10.33210/ca.v9i2.284

Carrillo, M. (2020). La Interculturalidad en la Educación Superior: El caso de Ecuador. Revista Polo Del Conocimiento, 5 (02), 715-731. https://doi.org/10.23857/pc.v5i2.1307

Cifuentes, J. (2020). Docencia online y Covid-19: la necesidad de reinventarse. Revista de Estilos de Aprendizaje, 13, 115-127. https://dialnet.unirioja.es/servlet/articulo?codigo=7681751\&orden=0\&info=link\%0Ahttps ://dialnet.unirioja.es/servlet/extart?codigo $=7681751$

Estela, R., Contreras, E., \& Incio, F. (2020). Enseñanza de la física universitaria durante la educación no presencial. Revista Epistemia, 4 (3), 152-165. https://doi.org/https://doi.org/10.26495/re.v4i3.1428

Gómez, R., Cano, J., \& Montoya, E. (2020). Método costeo ABC con simulación de Monte Carlo en la logística en la cadena de suministro en la industria 4.0. Cuadernos de Contabilidad, 21, 1-15. https://doi.org/https://doi.org/10.11144/Javeriana.cc21.mcas

Guajardo, C. (2020). Educación intercultural en escuelas multiculturales urbanas: estudio 
etnográfico en un centro escolar de la Ciudad de México. Boletín de Antropología. Universidad de Antioquia, Medellín, $35 \quad$ (59), 82-107. https://doi.org/http://dx.doi.org/10.17533/udea.boan.v35n59a06

Mera, L., Otero, P., \& Calle, V. (2021). Aplicación de la Simulación de Montecarlo para el Análisis de la Implementación de Estaciones de Carga Rápida para Vehículos Eléctricos en la Provincia de Galápagos. Revista Técnica "Energía," 2 (17), 143-152. https://doi.org/10.37116/revistaenergia.v17.n2.2021.434

Pamplona, J., Cuesta, J., \& Cano, V. (2019). Estrategias de enseñanza del docente en las áreas básicas: una mirada al aprendizaje escolar. Revista Eleuthera, 1 (21), 13-33. https://doi.org/10.17151/eleu.2019.21.2

Pardo, M., Marín, D., \& De Castro, A. (2021). Aprendizajes para la vida a través de la implementación de estrategias didácticas digitales: un estudio comparativo de casos. Revista Iberoamericana de Educación, $85 \quad$ (1), 17-38. https://doi.org/https://doi.org/10.35362/rie8514044

Parra, M. (2020). Actividades Lúdicas como Estrategias de Transición Educativa. Revista Scientific, 5 (17), 143-163. https://doi.org/https://doi.org/10.29394/Scientific.issn.25422987.2020.5.17.7.143-163

Pascual, J. (2008). EL BAMBÚ, UNA ALTERNATIVA SOSTENIBLE EN LA SOLUCIÓN DE LA VIVIENDA SOCIAL. Ciencia en su PC, (1), 89-99.

Prieto, G., \& Cabrera, D. (2020). Diseño y evaluación de una estrategia lúdica de aprendizaje para enseñar Simulación de Montecarlo. Espacios, 41 (13).

Ramírez, G. (2021). La familia y el juego como estrategia de aprendizaje a distancia durante la pandemia del Covid-19 en México: Una propuesta desde la enseñanza universitaria en ciencias de la salud. Revista de Educación a Distancia, 21 (65), 1-20. https://doi.org/https://doi.org/10.6018/red.456231

Rodríguez, J. (2006). El bambú como material de construcción. Conciencia Tecnológica, (31), 6769.

Ruíz, G. (2020). COVID-19: Pensar la educación en un escenario inédito. Revista Mexicana de Investigacion Educativa, $\quad 25 \quad$ (85), 229-237. http://www.scielo.org.mx/scielo.php?script=sci_arttext\&pid=S1405-66662020000200229

Ruiz-Quiroga, M. (2020). Formación de formadores en el marco de la educación intercultural: estado del arte. Hojas y Hablas, (19), 48-66. https://doi.org/10.29151/hojasyhablas.n19a3

Zavala, V., \& Franco, R. (2020). El Estado enseña sobre las lenguas originarias : una reflexión desde el programa "Aprendo en casa" (El Estado enseña sobre las lenguas originarias: una reflexión desde el programa "Aprendo en Casa"), 126. https://www.google.com/search?client=firefox-b- 
d\&q=El+Estado+enseña+sobre+las+lenguas+originarias\%3A+una+reflexión+desde+el+p rograma+"Aprendo+en+Casa" 